PALABRAS CLAVE

Exportaciones

Importaciones

Productos manufacturados

China

Análisis de datos

Modelos matemáticos

Estadísticas comerciales

Roberto Álvarez E.

Economista Senior,

División de Política Financiera,

Banco Central de Chile

œralvarez@bcentral.cl

Eugenio Figueroa B.

Profesor, Departamento de Economía,

Universidad de Chile

• efiguero@econ.uchile.cl

María Pía Figueroa Z

Investigadora Asociada

Departamento de Economía,

Universidad de Chile

œmfiguero@fen.uchile.cl

Macarena Palma E.

Investigadora Asociada,

Departamento de Economía,

Universidad de Chile

- mpalma@fen.uchile.cl

\section{Determinantes de las exportaciones mundiales de manufacturas a China, 1990-2006}

\author{
Roberto Alvarez E., Eugenio Figueroa B., \\ Maria Pía Figueroa Z. y Macarena Palma E.
}

$\mathrm{E}$

n este trabajo se estudian los factores determinantes de las exportaciones de manufacturas a China. Utilizando datos de 79 países correspondientes al período 1990-2006 y estimaciones de ecuaciones de gravedad, se analizan los efectos de la dotación de factores, las características geográficas y el grado de apertura de las economías. Los resultados, que son compatibles con el modelo de abundancia de factores, revelan que las economías de mayor dotación de capital humano exportan un mayor volumen de manufacturas a China. Asimismo, el tamaño de la economía y la proximidad geográfica con este país favorecen las exportaciones manufactureras. Los resultados no indican que otras características de los países, tales como la apertura comercial y la salida al mar, desempeñen un papel importante al respecto; tampoco la dotación de tierra por trabajador y de capital por trabajador Las implicaciones de este trabajo son útiles para las economías que procuran aprovechar el notable dinamismo de la economía china para diversificar sus exportaciones hacia los bienes manufacturados. 


\section{I}

\section{Introducción}

El marcado crecimiento económico experimentado por China en los últimos 20 años ha generado un gran aumento de la demanda de bienes y servicios extranjeros. Este país, que en el pasado tuvo una economía muy poco integrada, se ha convertido en uno de los principales actores del desarrollo económico mundial y en la actualidad representa aproximadamente el 15\% del comercio internacional de manufacturas (Lehmann, Moreno y Jaramillo, 2007). De mantenerse este dinamismo, se producirá un incremento sustancial de las exportaciones del resto del mundo hacia China, sobre todo de bienes en que su economía no tiene ventajas comparativas o no las desarrollará en el futuro.

En el contexto actual, las exportaciones de los países en desarrollo hacia este país se componen principalmente de materias primas, pero la gran magnitud de la economía china y las amplias expectativas de crecimiento pueden hacer de este un mercado bastante atractivo para la exportación de productos manufactureros. Con esta motivación, el objetivo del presente trabajo es analizar los factores determinantes de las exportaciones de manufacturas a China. Utilizando datos de 79 países correspondientes al período 1990-2006, primero se describen los principales hechos estilizados de las exportaciones manufactureras a este país y luego se utiliza un modelo de gravedad para estudiar sus factores determinantes. En particular, se analiza el rol que desempeñan al respecto la dotación de factores, las variables geográficas, la apertura al comercio internacional y el tamaño de la economía.
Ello permite identificar qué países, especialmente los menos desarrollados, tendrían mayores posibilidades de convertirse en importantes proveedores de las importaciones chinas de manufacturas.

Aunque existen diversos estudios sobre las consecuencias del ingreso de China al comercio mundial, ${ }^{1}$ hay pocos en que se aborde cómo las demás naciones en desarrollo podrían aprovechar esta oportunidad para cambiar su patrón de especialización hacia las manufacturas. Ello tiene especial importancia para las economías exportadoras de materias primas, que suelen verse afectadas por las fluctuaciones de la economía mundial y que podrían reducir esta vulnerabilidad si tuvieran una estructura exportadora más diversificada. En este sentido, el objetivo de nuestro trabajo es similar al de Mesquita Moreira (2007), quien identificó a China como un fuerte competidor de América Latina en el comercio de manufacturas. Pero, a diferencia del análisis realizado por este autor, exploramos si China puede convertirse en un mercado de destino importante para las exportaciones manufactureras y estudiamos una muestra más grande de países.

El presente trabajo se divide en cinco secciones. Después de esta introducción, se analiza la evolución de las exportaciones de manufacturas a China. En la tercera sección se describen las especificaciones y las fuentes de información, en la cuarta se presentan y discuten los resultados de las estimaciones del modelo de gravedad y en la última se ofrecen las conclusiones.

\section{II}

\section{Evolución de las exportaciones a China}

La irrupción de China en el escenario mundial ha sido de gran importancia debido a su dinámico desempeño económico, que se refleja en tasas de crecimiento anual

$\square$ Los autores agradecen los valiosos comentarios de un evaluador anónimo, así como las sugerencias recibidas durante un seminario de investigación del Departamento de Economía de la Universidad de Chile y la eficiente colaboración de Waldo Riveras. Los errores son de exclusiva responsabilidad de los autores. superiores al 9\% durante los últimos 17 años. Tras un prolongado período de aislamiento comercial, Deng Xiaoping impulsó un proceso de reformas económicas que culminó con una reducción significativa de

\footnotetext{
1 Al respecto, véanse los trabajos recientes de Blázquez-Lidoy, Rodríguez y Santiso (2006); Claro (2006); Devlin, Estevadeordal y Rodríguez-Clare (2006) y Rosales y Kuwayama (2007).
} 
las barreras al comercio. Esto se ha traducido en un incremento considerable del intercambio chino con el resto de mundo.

Como lo muestra el gráfico 1, en el período 19902006 las importaciones chinas aumentaron de 53.000 millones de dólares a poco más de 790.000 millones. Por otra parte, en el gráfico 2 se aprecia que las importaciones de manufacturas se incrementaron de 41.000 millones de dólares a poco más de 570.000 millones en el mismo período.

GRÁFICO 1

China: valor de las importaciones totales, 1990-2006

(En miles de millones de dólares)

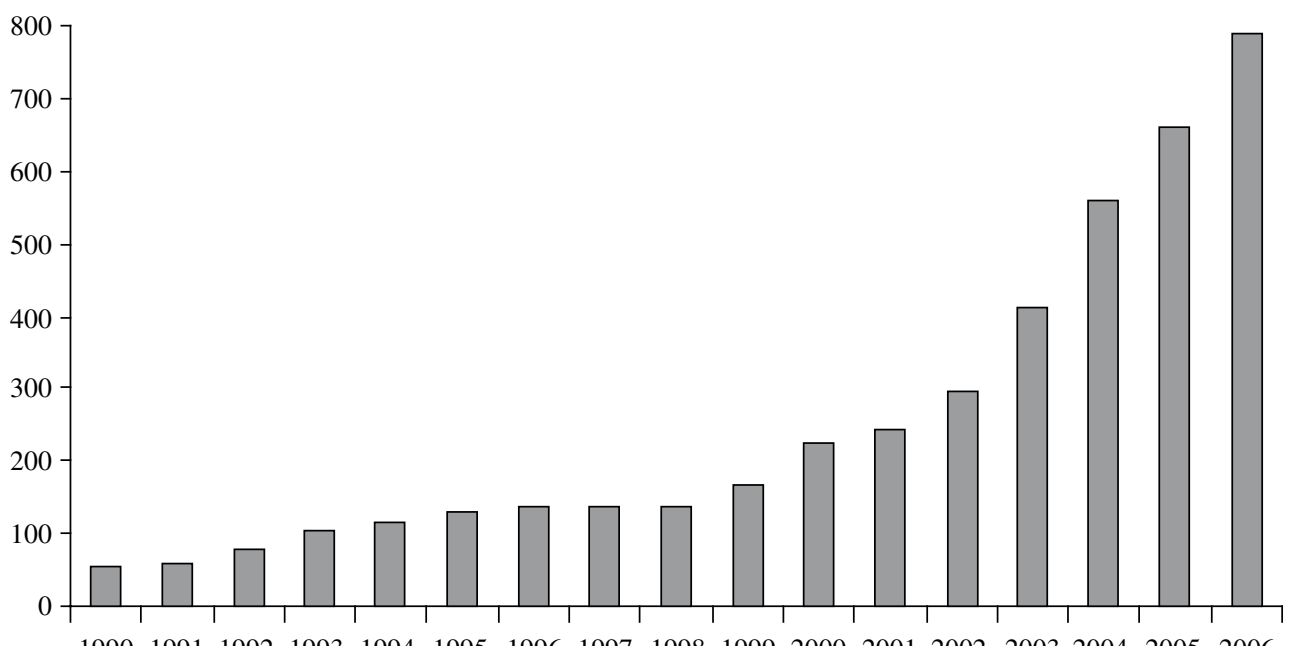

19901991199219931994199519961997199819992000200120022003200420052006

Fuente: elaboración propia a partir de cifras de la Base de datos estadísticos sobre el comercio de mercaderías (COMTRADE).

GRÁFICO 2

China: valor de las importaciones de manufacturas, 1990-2006

(En miles de millones de dólares)

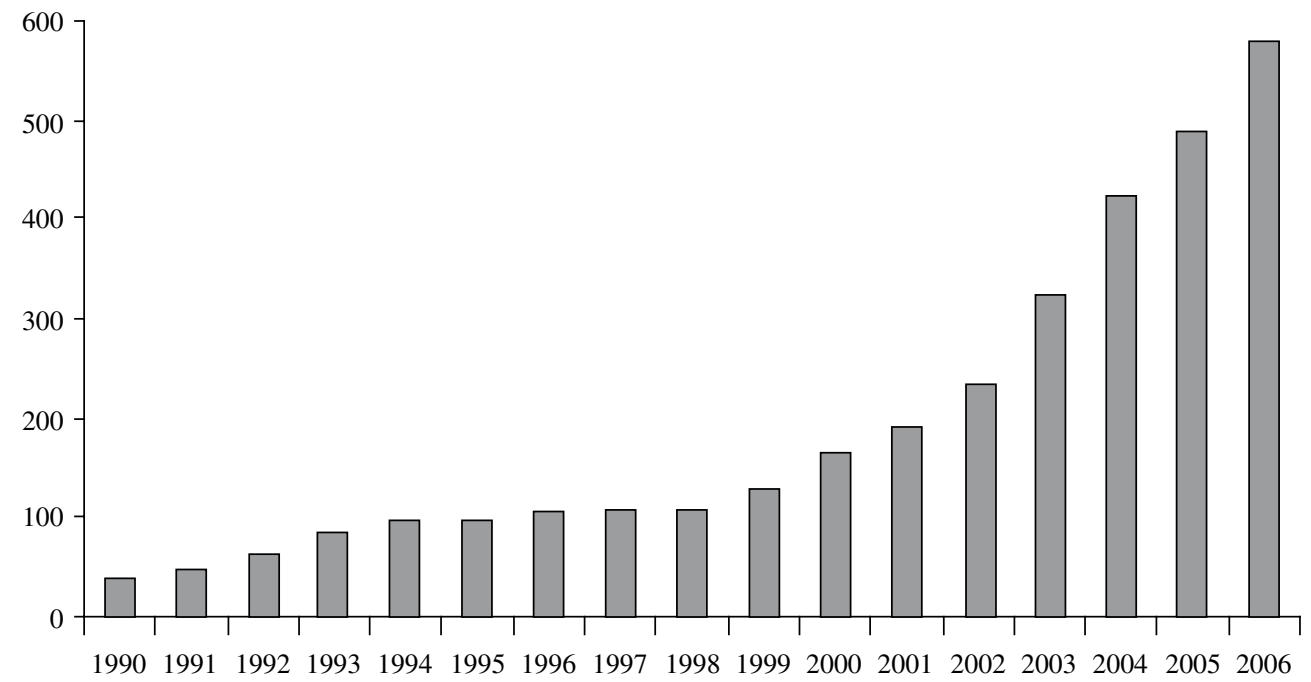

Fuente: elaboración propia a partir de cifras de la Base de datos estadísticos sobre el comercio de mercaderías (COMTRADE). 
Cabe preguntarse cómo se ha distribuido este incremento del comercio chino en las distintas regiones del mundo. El gráfico 3 muestra que, en general, todas han aprovechado las oportunidades de este mercado asiático, aumentando considerablemente el valor de sus exportaciones a China. Las exportaciones de América del Norte crecieron de 4.000 millones de dólares a 33.500 millones; las de Asia, de 800 a 21.000; las de Oceanía, de 700 a 10.300; las de Europa, de 400 a 5.800; las de África, de 20 a 300

\section{Exportaciones totales a China según regiones, 1990-2006}

(En miles de millones de dólares)

América Latina y el Caribe

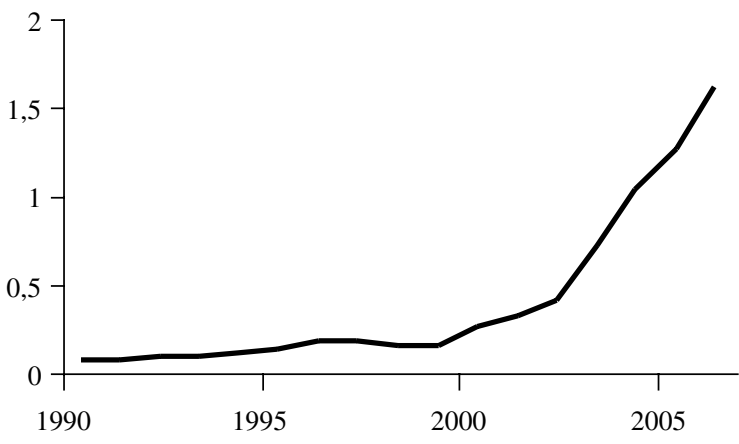

Europa

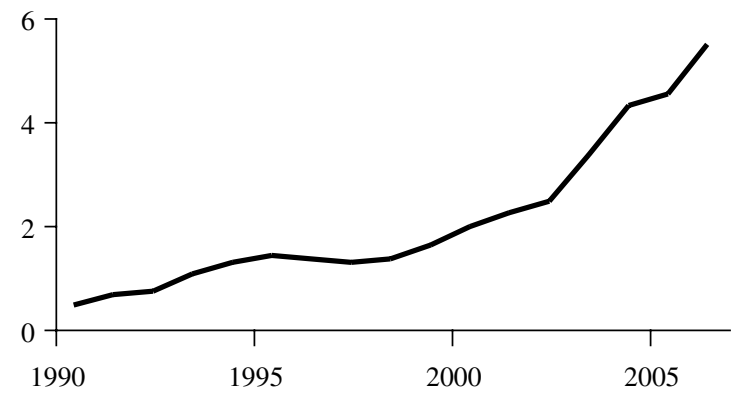

África

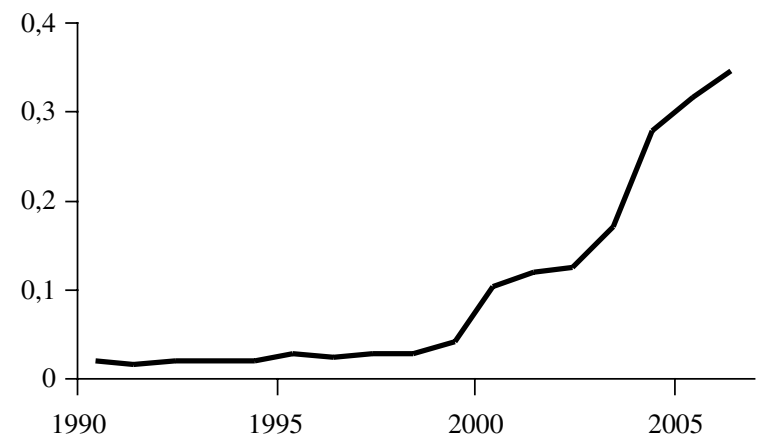

América del Norte

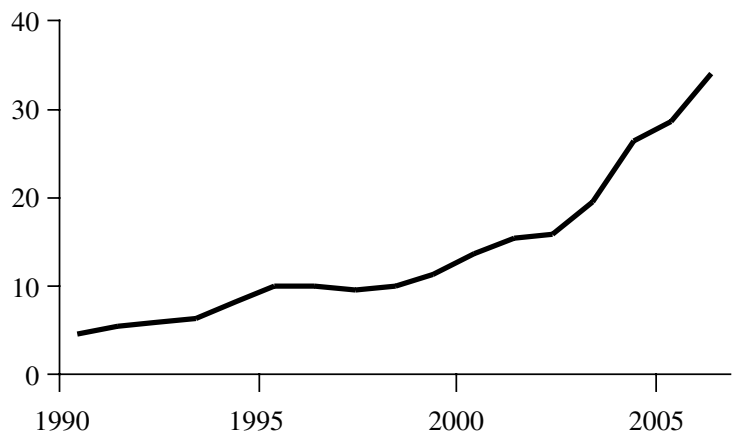

Asia

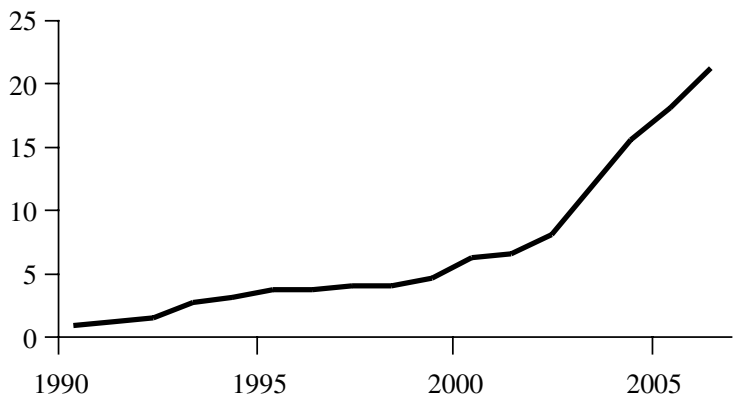

Oceanía

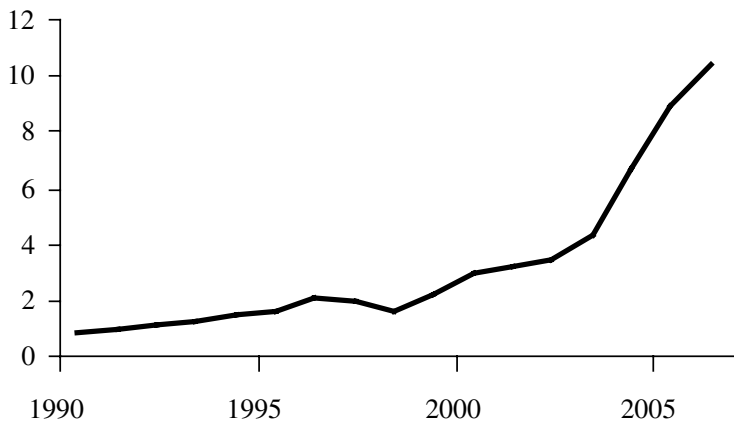

Fuente: elaboración propia a partir de cifras de la Base de datos estadísticos sobre el comercio de mercaderías (COMTRADE). 
y las de América Latina y el Caribe, de 100 a 1.600 millones de dólares. En el cuadro 1 se resumen los resultados antes mencionados.

Es interesante destacar la composición de las exportaciones a China, haciendo hincapié en la importancia relativa de los bienes manufacturados. ${ }^{2} \mathrm{El}$ gráfico 4 muestra la participación de las manufacturas en las exportaciones totales de cada región. Es posible apreciar que Europa es el continente en que esta alcanza el porcentaje más elevado, puesto que en el período 19902006 se incrementó un 82\% medio. También se observa un crecimiento significativo en el caso de Asia, donde las exportaciones de manufacturas a China aumentaron del 41\% del total en 1990 al 67\% en 2006; en América del Norte, ellas se incrementaron hasta alcanzar un $61 \%$ en 2006. La participación de bienes manufacturados en las exportaciones totales de Oceanía, África y América Latina y el Caribe aún no supera el $41 \%$, con la diferencia de que el porcentaje correspondiente a Oceanía ha

${ }^{2}$ La clasificación de bienes manufacturados utilizada en esta sección y en el resto del trabajo corresponde a la realizada por Leamer (1984), que se muestra detalladamente en el anexo. ido aumentando, mientras que el de África y América Latina y el Caribe se ha reducido.

En general, la evolución de las exportaciones de manufacturas a China parece ser compatible con la idea de que los países más ricos —esto es, de capital físico y humano más abundante- - suelen tener ventajas comparativas en la producción de bienes manufacturados, tal como lo prevé el modelo tradicional de Heckscher-Ohlin (Leamer, 1995). También indicaría que los países grandes presentan un patrón de exportaciones especializado en las manufacturas, como sería el caso de los países europeos y de América del Norte (Perkins y Syrquin, 1989).

En el cuadro 2 se muestra más detalladamente la participación de los países de América Latina y el Caribe en las importaciones chinas de manufacturas. Las cifras revelan claramente que las economías de la región en su conjunto representan una proporción bastante baja y decreciente del total. Esto incluye a las economías más grandes, cuya participación en las importaciones chinas de bienes manufacturados también se redujo en la década de 1990. La del Brasil disminuyó del 0,74\% al $0,43 \%$, la de la Argentina del $0,36 \%$ al $0,09 \%$ y la de México del $0,34 \%$ al $0,31 \%$, para luego remontar hasta un $0,53 \%$ en 2006.

CUADRO 1

Evolución de las exportaciones a China, 1990-2006

\begin{tabular}{|c|c|c|c|c|c|c|c|c|}
\hline Región & $\begin{array}{r}\text { (en mil } \\
d \epsilon\end{array}$ & $\begin{array}{l}1990 \\
\text { es de millones } \\
\text { dólares) }\end{array}$ & ${ }_{d}^{(e n ~ m i}$ & $\begin{array}{l}2006 \\
\text { s de millones } \\
\text { dólares) }\end{array}$ & $\begin{array}{r}\text { Variac } \\
1 \\
\text { (en }\end{array}$ & $\begin{array}{l}\text { ón porcentual } \\
90-2006 \\
\text { orcentajes) }\end{array}$ & $\begin{array}{r}\text { Tasa d } \\
\text { (en } 1\end{array}$ & $\begin{array}{l}\text { crecimiento } \\
\text { anual }^{\mathrm{a}} \\
\text { orcentajes }^{2}\end{array}$ \\
\hline América del Norte & & 4,0 & & 33,5 & & 738 & & 13,89 \\
\hline Asia & & 0,8 & & 21,0 & & 2525 & & 20,38 \\
\hline Oceanía & & 0,7 & & 10,3 & & 1371 & & 19,31 \\
\hline Europa & & 0,4 & & 5,8 & & 1350 & & 17,86 \\
\hline África & & 0,02 & & 0,3 & & 1400 & & 20,53 \\
\hline América Latina y el Caribe & & 0,1 & & 1,6 & & 1500 & & 19,61 \\
\hline \multirow{2}{*}{ Total países } & Totales & Manufacturas & Totales & Manufacturas & Totales & Manufacturas & Totales & Manufacturas \\
\hline & 53 & 41 & 790 & 570 & 1391 & 1290 & 16,35 & 15,59 \\
\hline
\end{tabular}

Fuente: elaboración propia a partir de cifras de la Base de datos estadísticos sobre el comercio de mercaderías (COMTRADE).

a Corresponde a la media geométrica de las tasas de crecimiento anual durante el período 1990-2006. 
GRÁFICO 4

Exportaciones de manufacturas a China según regiones, 1990-2006 (En porcentajes)

América Latina y el Caribe

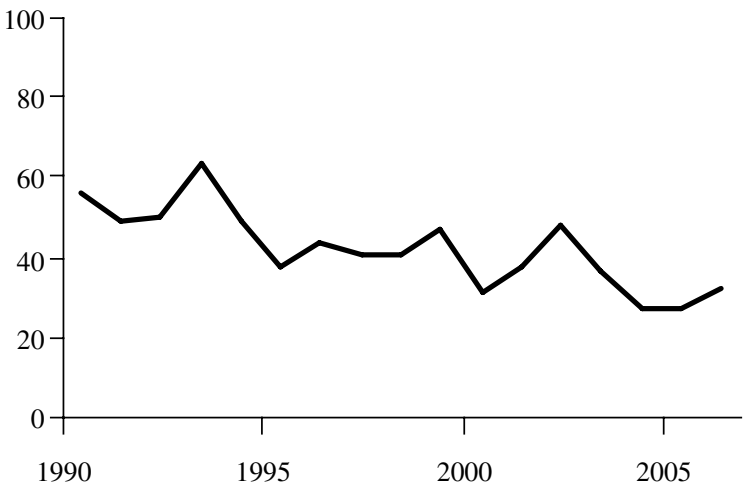

Europa

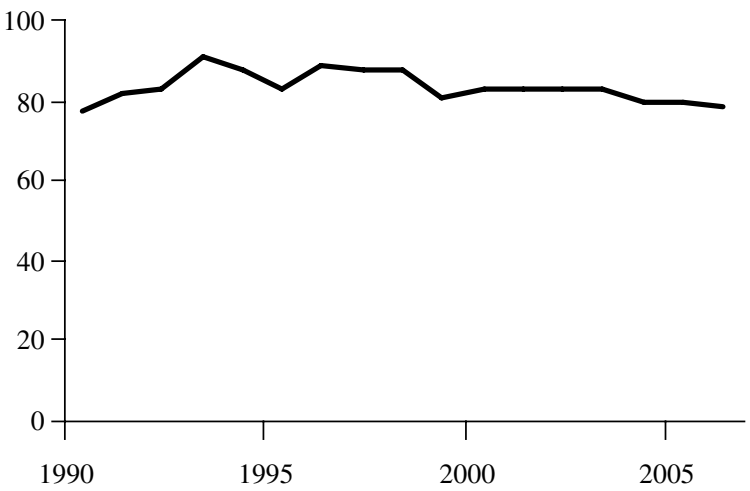

África

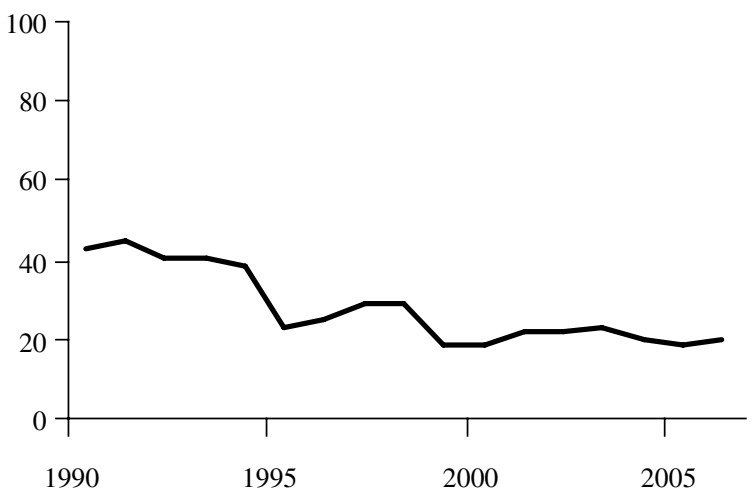

América del Norte

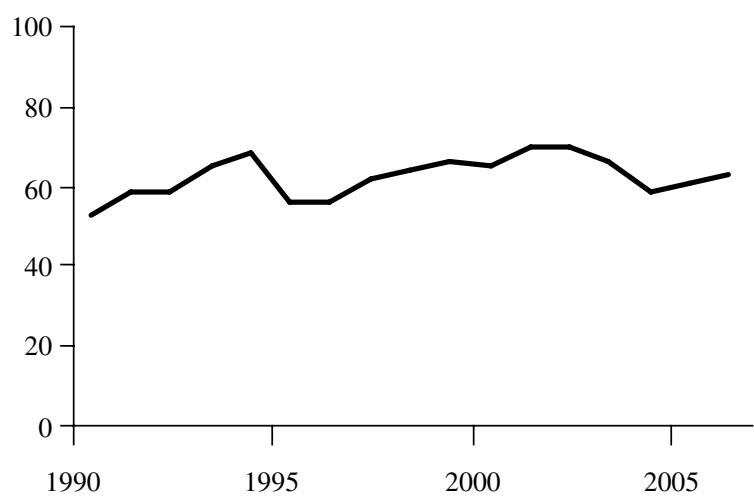

Asia

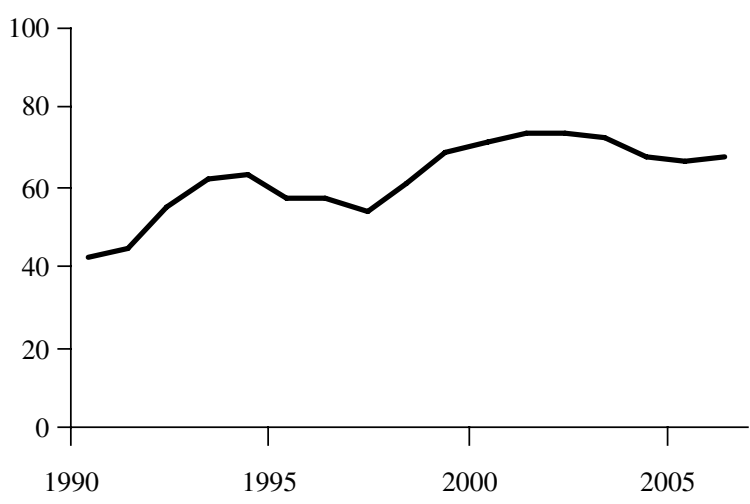

Oceanía

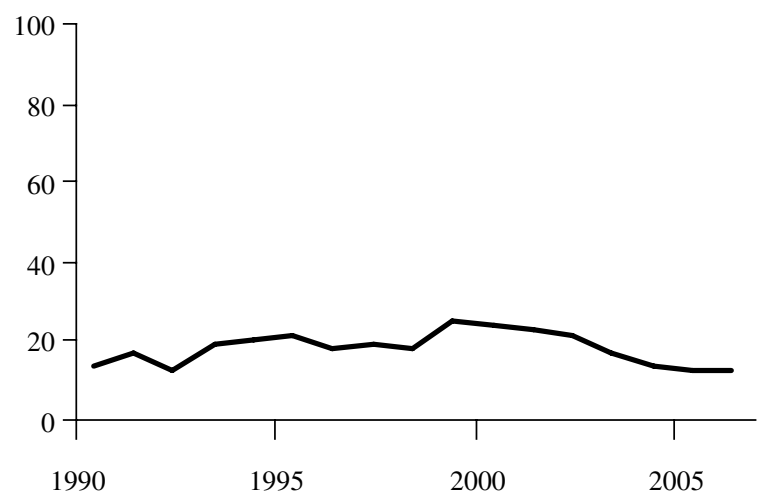

Fuente: elaboración propia a partir de cifras de la Base de datos estadísticos sobre el comercio de mercaderías (COMTRADE). 
CUADRO 2

América Latina y el Caribe (países seleccionados): participación en las importaciones chinas de manufacturas, 1990-2006

(En porcentajes medios del período)

\begin{tabular}{|c|c|c|c|c|c|c|}
\hline \multirow{2}{*}{ País } & \multicolumn{6}{|c|}{ Períodos } \\
\hline & 1990-1992 & 1993-1995 & 1966-1998 & 1999-2001 & 2002-2004 & $2005-2006$ \\
\hline Argentina & 0,36 & 0,18 & 0,15 & 0,13 & 0,14 & 0,09 \\
\hline Bolivia, Estado Pl. de & 0,00 & 0,00 & 0,00 & 0,00 & 0,00 & 0,00 \\
\hline Brasil & 0,74 & 0,68 & 0,28 & 0,29 & 0,58 & 0,43 \\
\hline Chile & 0,02 & 0,01 & 0,03 & 0,03 & 0,03 & 0,05 \\
\hline Colombia & 0,00 & 0,00 & 0,00 & 0,01 & 0,02 & 0,05 \\
\hline Costa Rica & 0,00 & 0,00 & 0,00 & 0,01 & 0,19 & 0,35 \\
\hline Ecuador & 0,00 & 0,00 & 0,00 & 0,00 & 0,00 & 0,00 \\
\hline El Salvador & 0,00 & 0,00 & 0,00 & 0,00 & 0,00 & 0,00 \\
\hline Guatemala & 0,00 & 0,00 & 0,00 & 0,00 & 0,00 & 0,00 \\
\hline Guyana & 0,00 & 0,00 & 0,00 & 0,00 & 0,00 & 0,00 \\
\hline Honduras & 0,00 & 0,00 & 0,00 & 0,00 & 0,00 & 0,00 \\
\hline Jamaica & 0,00 & 0,00 & 0,00 & 0,00 & 0,00 & 0,00 \\
\hline México & 0,34 & 0,11 & 0,14 & 0,31 & 0,59 & 0,53 \\
\hline Nicaragua & 0,00 & 0,00 & 0,00 & 0,00 & 0,00 & 0,00 \\
\hline Panamá & 0,00 & 0,01 & 0,00 & 0,00 & 0,00 & 0,00 \\
\hline Paraguay & 0,00 & 0,00 & 0,00 & 0,00 & 0,00 & 0,00 \\
\hline Perú & 0,01 & 0,00 & 0,01 & 0,00 & 0,00 & 0,01 \\
\hline Rep. Dominicana & 0,00 & 0,00 & 0,00 & 0,00 & 0,00 & 0,01 \\
\hline Trinidad y Tabago & 0,01 & 0,00 & 0,00 & 0,00 & 0,00 & 0,00 \\
\hline Uruguay & 0,27 & 0,08 & 0,07 & 0,06 & 0,03 & 0,02 \\
\hline Venezuela, Rep. B. de & 0,05 & 0,01 & 0,01 & 0,01 & 0,05 & 0,04 \\
\hline Total & 1,80 & 1,08 & 0,69 & 0,85 & 1,63 & 1,58 \\
\hline
\end{tabular}

Fuente: elaboración propia a partir de cifras de la Base de datos estadísticos sobre el comercio de mercaderías (COMTRADE).

\section{III}

\section{Análisis empírico}

\section{Especificación del modelo}

Una de las metodologías más utilizadas y exitosas para estudiar los factores que determinan el intercambio de bienes y su importancia cuantitativa es el denominado modelo de gravedad. Propuesto inicialmente por Tinbergen (1962), este modelo ha tenido amplia aplicación para responder preguntas relativas al efecto de los acuerdos preferenciales en el comercio bilateral (Baier y Bergstrand, 2007), las repercusiones del ingreso de los países a la Organización Mundial del Comercio (Rose, 2004) y las causas determinantes del comercio electrónico (Blum y Goldfarb, 2006), entre otras.

La ecuación básica a estimar está dada por:

$$
X_{i j}=a_{0}\left(P I B_{i}\right)^{a_{1}}\left(P I B_{j}\right)^{a_{2}}\left(D I S T_{i j}\right)^{a_{3}} e^{a_{4} V E C_{i j}+a_{5} L E N G_{i j}} \varepsilon_{i j}
$$

donde $X_{i j}$ son las exportaciones del país $i$ al país $j, P I B_{i}$ es el producto del país $i$, DIST es la distancia entre ambos países y VEC y LENG son variables categóricas de los países que tienen una frontera y un lenguaje comunes.

Tomando el logaritmo a ambos lados de la ecuación (1), se obtiene:

$$
\begin{gathered}
\ln \left(X_{i j}\right)=a_{0}+a_{1} \ln \left(P I B_{i}\right)+a_{2} \ln \left(P I B_{j}\right)+a_{3} \\
\ln \left(D I S T_{i j}\right)+a_{4} V E C+a_{5} L E N G+\varepsilon_{i j}
\end{gathered}
$$

En este trabajo se analiza el comercio con China de 79 países de los cuales se dispone de información sobre el período 1990-2006 (véase el listado de países del anexo 2). El modelo de gravedad puede ampliarse para determinar en qué medida las exportaciones de manufacturas a China obedecen a factores geográficos que no varían en el tiempo — por ejemplo, distancia y 
frontera común-y a otras características que sí lo hacen - tamaño del país de que se trate, dotación de factores y política comercial-.

Para analizar los efectos de otras variables geográficas, de la dotación de factores y de las barreras arancelarias al comercio, se amplió la ecuación (2) y se agregó una variable categórica correspondiente a los países exportadores que tienen salida al mar. En cuanto a la dotación de factores, se incluyó una estimación del capital humano, el capital físico y los recursos naturales. Para medir los dos primeros se usó la escolaridad de la población mayor de 15 años y la razón de capital por trabajador, respectivamente, del país exportador. Siguiendo a Leamer (1987) y Schott (2003), como medida de la abundancia de recursos naturales se utilizó la superficie de tierra arable por trabajador. Las barreras al comercio corresponden al arancel medio a las importaciones de cada país exportador, ${ }^{3}$ variable que no refleja las barreras que China impone a las exportaciones de otros países, sino más bien el grado de apertura comercial del país de que se trate.

Las variables escolaridad, capital por trabajador y abundancia de recursos naturales se incluyeron siguiendo las implicaciones del modelo de Heckscher-Ohlin, esto es, que un país determinado tiene ventajas comparativas en los productos de uso intensivo de su factor productivo más cuantioso. Según el teorema de Rybczynski, suponiendo que las manufacturas son de alta densidad de capital, las diferencias en materia de capital humano y físico deberían reflejarse en el volumen de exportaciones manufactureras a China. En otras palabras, los países de mayor capital deberían exportar más manufacturas a este país. Ampliando el modelo básico, Leamer (1987) demostró que la abundancia de recursos naturales afecta la senda de desarrollo de las economías, haciendo menos probable que ellas tengan ventajas comparativas en la producción de bienes manufacturados. Por este motivo, se incluyó como control la variable superficie de tierra por trabajador.

Los signos previstos de todas las variables explicativas son positivos, con excepción de distancia, arancel medio y abundancia de recursos naturales. Esto porque se prevé que un PIB más elevado, un lenguaje y una frontera comunes, el hecho de que el país exportador tenga salida al mar, una mayor escolaridad de la

\footnotetext{
${ }^{3}$ Todas las variables mencionadas se midieron en logaritmos.
}

fuerza de trabajo y un mayor nivel de capital físico por trabajador contribuyen a aumentar las exportaciones de manufacturas.

De un total de 1.343 observaciones posibles en los 79 países, el resultado de 123 de ellas en materia de exportaciones a China fue igual a cero. Dado que al aplicar logaritmos estas observaciones serían omitidas, perdiéndose así información relevante, se utilizó la alternativa que se emplea comúnmente de definir la variable dependiente como $\ln (1+\mathrm{X})$.

\section{Fuentes de información}

Los datos sobre intercambio comercial provienen de la Base de datos estadísticos sobre el comercio de mercaderías (COMTRADE), ${ }^{4}$ que contiene información detallada del comercio bilateral (exportaciones e importaciones) por industria en subgrupos de cuatro dígitos según la Clasificación Uniforme para el Comercio Internacional (CUCI) (Rev. 2). Dado que nuestro trabajo está centrado en las exportaciones de manufacturas durante el período $1990-2006,{ }^{5}$ se utilizó la clasificación de Leamer (1984) de productos manufacturados. Las corrientes de comercio expresadas en dólares nominales, siguiendo a Rose (2004), se deflactaron de acuerdo con el índice de precios al por mayor de los Estados Unidos.

En cuanto a la dotación de factores de los distintos países, se emplearon diversas fuentes de información. Los datos de lenguaje, distancia, salida al mar y contigüidad provienen del Centre d'études prospectives et d'informations internationales (CEPI) ${ }^{6}$ La información sobre el PIB y la tierra agrícola se tomó de los Indicadores del desarrollo mundial del Banco Mundial, mientras que la de escolaridad y capital por trabajador es de Bosworth y Collins (2003). Finalmente, los datos sobre aranceles a las importaciones, que se utilizaron como medida del grado de apertura comercial, provienen del Banco Mundial.

Las estadísticas descriptivas de todas las variables utilizadas en las estimaciones se muestran en el cuadro 3 .

\footnotetext{
${ }^{4}$ Véase [en línea] comtrade.un.org.

${ }^{5}$ Hay datos disponibles a partir de 1962, pero el período anterior a 1990 es menos relevante de analizar debido a la escasa magnitud de las corrientes de comercio con China.

${ }^{6}$ Véase [en línea] http://www.cepii.org/anglaisgraph/bdd/bdd.htm
} 
CUADRO 3

Estadísticas descriptivas de las variables explicativas

\begin{tabular}{|c|c|c|c|c|c|}
\hline Variable & Observaciones & Media & Desv. est. & Mínimo & Máximo \\
\hline Ln (1 + exportaciones) & 1343 & 8,77 & 5,00 & 0 & 18,17 \\
\hline Ln (distancia) & 1343 & 9,11 & 0,54 & 6,91 & 9,86 \\
\hline Mar & 1343 & 0,86 & 0,35 & 0 & 1 \\
\hline Contigüidad & 1343 & 0,03 & 0,16 & 0 & 1 \\
\hline Lenguaje común & 1343 & 0,03 & 0,16 & 0 & 1 \\
\hline Ln (tierra arable) & 1343 & $-0,9$ & 1,12 & $-7,68$ & 2,06 \\
\hline $\operatorname{Ln}(\mathrm{PIB})$ & 1264 & 24,52 & 2,01 & 19,91 & 30,03 \\
\hline Escolaridad & 1343 & 6,62 & 2,83 & 0,79 & 12,12 \\
\hline Ln (capital por trabajador) & 1343 & $-5,07$ & 2,77 & $-13,82$ & $-1,2$ \\
\hline Arancel medio & 1343 & 13,02 & 10,28 & 0 & 94 \\
\hline
\end{tabular}

Fuente: Banco Mundial, Centre d'études prospectives et d'informations internationales (CEPII), B. Bosworth y S. M. Collins, "The empirics of growth: an update", Brookings Papers on Economic Activity, vol. 34, № 2, Washington, D.C., The Brookings Institution, 2003, y Base de datos estadísticos sobre el comercio de mercaderías (COMTRADE).

\section{IV}

\section{Resultados de las estimaciones}

En esta sección se describen los resultados de las estimaciones del modelo de gravedad. Las estimaciones de corte transversal correspondientes a diferentes períodos de tiempo se presentan en el cuadro 4. Para reducir los efectos de las oscilaciones anuales — por ejemplo, variación de precios de algunos productos o perturbaciones transitorias - en la calidad de las estimaciones, se utilizaron intervalos de tres años. Se descartó la alternativa de realizar las estimaciones a partir de un panel de datos, ya que algunas variables de interés como la distancia y el lenguaje común no varían a lo largo del tiempo y serían absorbidas por un efecto fijo del país exportador.

La variable que mide la distancia entre el exportador y China es negativa y estadísticamente significativa en todas las especificaciones, y el valor del parámetro oscila entre $-1,98$ y $-1,47$. Como era de prever, una mayor distancia con China incrementa los costos del comercio, lo que se traduce en una reducción de las exportaciones a esta economía. Para tener una idea cuantitativa de la importancia que según estas estimaciones tiene la distancia, se calculó la diferencia entre las exportaciones de un país de América Latina y otro de Asia, usando la distancia media de cada grupo con China y el parámetro correspondiente al último período estimado. En este caso, la diferencia en materia de exportaciones está dada por:

$$
\partial \operatorname{Ln}(X)=-1,47\left[\ln D_{A L}-\ln D_{A S I A}\right]=1,91
$$

Esto significa que si el país medio de América Latina estuviera a la misma distancia de China que el país medio de Asia, podría incrementar sus exportaciones más del $190 \%$.

De acuerdo con lo previsto, la existencia de un lenguaje común con China ejerce una influencia positiva y su contribución es significativamente distinta de cero a partir del segundo período de análisis. Estos resultados son compatibles con los encontrados en estudios anteriores en el sentido de que los países que tienen el mismo idioma comercian más entre sí (Rose, 2004).

La variable contigüidad no es significativa en ninguno de los años estudiados. Su signo previsto era positivo, ya que el menor costo del intercambio comercial entre los países colindantes suele favorecer el comercio recíproco. Sin embargo, solo dos de los 79 países de la muestra tienen una frontera común con China: la India y Pakistán. Otro factor geográfico que al parecer no afecta las exportaciones de manufacturas es el hecho de que el país exportador tenga salida al mar.

Los resultados indican que la abundancia de recursos naturales, medida en este caso según la razón entre tierra y trabajo, no parece afectar significativamente las exportaciones de manufacturas a China en ninguno de los años estudiados. Si bien es posible que haya variables más adecuadas para medir la dotación de recursos naturales, no había una muestra de países en que estuvieran disponibles durante el período de tiempo analizado en este trabajo. 
CUADRO 4

Estimaciones de corte transversal, 1990-2006

\begin{tabular}{|c|c|c|c|c|c|c|}
\hline Variables & 1990-1992 & 1993-1995 & 1996-1998 & 1999-2001 & 2002-2004 & 2005-2006 \\
\hline Ln (distancia) & $\begin{array}{r}-1,781^{\mathrm{a}} \\
(0,529)\end{array}$ & $\begin{array}{r}-1,980^{\mathrm{a}} \\
(0,430)\end{array}$ & $\begin{array}{r}-1,981^{\mathrm{a}} \\
(0,409)\end{array}$ & $\begin{array}{r}-1,876^{\mathrm{a}} \\
(0,417)\end{array}$ & $\begin{array}{r}-1,511^{\mathrm{a}} \\
(0,481)\end{array}$ & $\begin{array}{c}-1,469^{\mathrm{a}} \\
(0,535)\end{array}$ \\
\hline Lenguaje común & $\begin{array}{c}2,083 \\
(1,279)\end{array}$ & $\begin{array}{c}2,104^{\mathrm{b}} \\
(0,997)\end{array}$ & $\begin{array}{r}2,764^{\mathrm{a}} \\
(0,829)\end{array}$ & $\begin{array}{r}3,310^{\mathrm{a}} \\
(0,493)\end{array}$ & $\begin{array}{r}3,571^{\mathrm{a}} \\
(0,474)\end{array}$ & $\begin{array}{r}3,557^{\mathrm{a}} \\
(0,511)\end{array}$ \\
\hline Contigüidad & $\begin{array}{c}0,734 \\
(1,454)\end{array}$ & $\begin{array}{c}1,425 \\
(1,135)\end{array}$ & $\begin{array}{l}2,812^{\mathrm{c}} \\
(1,416)\end{array}$ & $\begin{array}{c}0,908 \\
(1,600)\end{array}$ & $\begin{array}{c}0,817 \\
(1,476)\end{array}$ & $\begin{array}{l}0,00422 \\
(1,406)\end{array}$ \\
\hline Mar & $\begin{array}{c}-0,135 \\
(1,053)\end{array}$ & $\begin{array}{c}0,392 \\
(0,758)\end{array}$ & $\begin{array}{c}-0,259 \\
(0,723)\end{array}$ & $\begin{array}{c}-0,455 \\
(0,693)\end{array}$ & $\begin{array}{c}-0,774 \\
(0,704)\end{array}$ & $\begin{array}{c}-0,650 \\
(0,686)\end{array}$ \\
\hline Ln (tierra arable por trab.) & $\begin{array}{c}0,163 \\
(0,260)\end{array}$ & $\begin{array}{c}0,218 \\
(0,215)\end{array}$ & $\begin{array}{c}0,211 \\
(0,208)\end{array}$ & $\begin{array}{c}0,365 \\
(0,360)\end{array}$ & $\begin{array}{c}0,282 \\
(0,388)\end{array}$ & $\begin{array}{c}0,155 \\
(0,410)\end{array}$ \\
\hline Ln (PIB) & $\begin{array}{r}1,288^{\mathrm{a}} \\
(0,175)\end{array}$ & $\begin{array}{r}1,339^{\mathrm{a}} \\
(0,153)\end{array}$ & $\begin{array}{r}1,289^{\mathrm{a}} \\
(0,164)\end{array}$ & $\begin{array}{l}1,581^{\mathrm{a}} \\
(0,150)\end{array}$ & $\begin{array}{r}1,528^{\mathrm{a}} \\
(0,148)\end{array}$ & $\begin{array}{l}1,618^{\mathrm{a}} \\
(0,154)\end{array}$ \\
\hline Ln (escolaridad) & $\begin{array}{c}0,302 \\
(1,165)\end{array}$ & $\begin{array}{r}1,731^{\mathrm{b}} \\
(0,793)\end{array}$ & $\begin{array}{r}2,308^{\mathrm{a}} \\
(0,712)\end{array}$ & $\begin{array}{r}2,098^{\mathrm{a}} \\
(0,751)\end{array}$ & $\begin{array}{r}2,274^{\mathrm{a}} \\
(0,717)\end{array}$ & $\begin{array}{r}1,264^{\mathrm{b}} \\
(0,540)\end{array}$ \\
\hline Ln (capital por trabajador) & $\begin{array}{c}0,253 \\
(0,221)\end{array}$ & $\begin{array}{c}0,0499 \\
(0,149)\end{array}$ & $\begin{array}{r}0,0577 \\
(0,150)\end{array}$ & $\begin{array}{c}-0,0218 \\
(0,132)\end{array}$ & $\begin{array}{c}-0,0282 \\
(0,174)\end{array}$ & $\begin{array}{c}-0,0453 \\
(0,162)\end{array}$ \\
\hline Ln (arancel medio) & $\begin{array}{c}0,0182 \\
(0,567)\end{array}$ & $\begin{array}{c}-0,372 \\
(0,387)\end{array}$ & $\begin{array}{c}-0,444 \\
(0,347)\end{array}$ & $\begin{array}{c}0,326 \\
(0,456)\end{array}$ & $\begin{array}{c}0,389 \\
(0,397)\end{array}$ & $\begin{array}{c}0,313 \\
(0,418)\end{array}$ \\
\hline Constante & $\begin{array}{l}-5,890 \\
(8,942)\end{array}$ & $\begin{array}{c}-8,099 \\
(7,239)\end{array}$ & $\begin{array}{c}-7,590 \\
(7,072)\end{array}$ & $\begin{array}{c}-16,81^{\mathrm{a}} \\
(6,122)\end{array}$ & $\begin{array}{r}-18,17^{\mathrm{a}} \\
(6,255)\end{array}$ & $\begin{array}{r}-18,68^{a} \\
(6,262)\end{array}$ \\
\hline Observaciones & 79 & 79 & 78 & 77 & 77 & 77 \\
\hline $\mathrm{R}^{2}$ & 0,687 & 0,810 & 0,817 & 0,777 & 0,754 & 0,730 \\
\hline
\end{tabular}

Fuente: elaboración propia.

Nota: Errores estándar sólidos entre paréntesis.

a $\mathrm{p}<0.01,{ }^{\mathrm{b}} \mathrm{p}<0.05,{ }^{\mathrm{c}} \mathrm{p}<0.1$

El tamaño del país exportador, medido según el PIB, es positivo y estadísticamente significativo en todos los períodos estudiados. Este efecto tamaño podría obedecer a la presencia de economías de escala en la producción de manufacturas (Perkins y Syrquin, 1989), pero también al hecho de que los países más grandes tienden a exportar un mayor volumen de todo tipo de bienes (Hanson y Xiang, 2004). A fin de contextualizar los resultados sobre el tamaño de la economía, cabe considerar dos países como Trinidad y Tabago y el Brasil que se cuentan entre los que pertenecen al $10 \%$ más pequeño y al $10 \%$ más grande de la muestra utilizada, respectivamente. A partir de las estimaciones del último período, la diferencia porcentual entre ambos en materia de exportaciones de manufacturas estaría dada por:

$$
\partial \operatorname{Ln}(X)=1,618\left[\ln \left(P I B_{B r a s i l}\right)-\operatorname{Ln}\left(P I B_{T y T}\right]=6.53\right.
$$

Estas cifras indican que el tamaño de la economía se traduce en marcadas diferencias en cuanto a capacidad de exportar bienes manufacturados.

En general, los resultados parecen ser bastante compatibles con la teoría tradicional del comercio internacional. De hecho, la variable que mide la abundancia de capital humano se relaciona positivamente con las exportaciones de manufacturas. La escolaridad de la fuerza de trabajo es positiva y significativa, con excepción del primer período estudiado. En este caso, las diferencias en materia de exportaciones pueden ilustrarse sobre la base de la escolaridad media de los países latinoamericanos y europeos. Dado que los parámetros tienden a variar de un período en otro y que en algunos casos no son significativos, se utilizó la media de los cuatro períodos analizados. Los resultados indican que si el país medio de América Latina tuviera la misma escolaridad del país medio de Europa, sus exportaciones se incrementarían casi un 35\%. Por otra parte, la variable capital por trabajador resultó ser no significativa en todos los períodos estudiados.

Finalmente, los resultados sobre apertura comercial, medida según el arancel medio a las importaciones, no indican que las economías más abiertas tiendan a exportar un mayor volumen de bienes manufacturados a China. Naturalmente, estos resultados deben analizarse con cuidado. De partida, en las estimaciones no se consideraron otras barreras 
al comercio como las restricciones no arancelarias, ya que no estaban disponibles en la muestra de países utilizada. Sin embargo, dado que la mayoría de los países ha abierto su economía durante las últimas décadas, este resultado puede ser compatible con la idea de que las barreras arancelarias ya no constituyen

\section{$\mathrm{V}$}

\section{Conclusiones}

El crecimiento económico de China y el rol significativo que ha adquirido como amplio consumidor de materias primas y de bienes más elaborados como las manufacturas ha abierto grandes posibilidades a los países exportadores de todo el mundo y representa un potencial de creciente importancia para los países en desarrollo cuya estrategia de crecimiento se basa en el aprovechamiento de las ventajas comparativas y la inserción en los mercados internacionales.

El propósito de este trabajo ha sido identificar los factores determinantes de las exportaciones de manufacturas a China, utilizando un modelo de gravedad al que se incorporaron variables relativas a las condiciones geográficas de los países, a la dotación de factores y a la política comercial.

Los resultados revelan la importancia de tres elementos fundamentales: la distancia entre el país exportador y China, su dotación de factores y el tamaño de la economía. Dado que este último componente es relativamente exógeno de las políticas que pueden aplicar las autoridades económicas, la discusión de los resultados se centra sobre todo en los aspectos relacionados con la distancia y la dotación de factores.

Pese a que la distancia es también un factor exógeno de la economía, existen políticas que pueden reducir sus efectos negativos en el comercio y específicamente en las exportaciones de manufacturas. De hecho, considerando las repercusiones negativas de los costos de transporte en el comercio, los países que están más alejados de China y otros centros del intercambio mundial tienen una desventaja natural que deben compensar mediante el mejoramiento de la infraestructura pertinente. En este caso, el desafío de las economías latinoamericanas es aún mayor que el de aquellas que poseen una ubicación más privilegiada. Las políticas públicas orientadas a perfeccionar la infraestructura de carreteras, sobre todo las que conectan los centros de producción de manufacturas con los puertos de salida de las exportaciones, así como a un impedimento significativo contra las exportaciones de manufacturas. De hecho, Wacziarg y Horn-Welch (2003) muestran que entre 1990 y 2000 el porcentaje de economías que pueden calificarse como abiertas al comercio internacional se incrementó de algo más del $40 \%$ al $73 \%$. mejorar el equipamiento y a modernizar y mecanizar los puertos y aeropuertos, podrían tener efectos importantes al respecto. Por otra parte, la adopción de una política de concesiones para realizar las obras públicas necesarias permitiría evitar las restricciones de capitales de inversión que son particularmente limitados en algunos países de la región. Asimismo, convendría aplicar políticas encaminadas a mejorar la eficiencia y a eliminar la corrupción de los sistemas aduaneros y los mecanismos de certificación de las exportaciones que a veces representan un costo adicional para los exportadores. En este sentido, las políticas y programas orientados a aumentar la transparencia de los servicios públicos, capacitar a los funcionarios estatales y promover y crear servicios públicos profesionalizados, con carreras funcionarias basadas en la calificación y el mérito e independientes de las influencias políticas, representan una vía de solución de muchos de los problemas de ineficacia que se traducen en altos costos para los exportadores. En los países en que se ha registrado un aumento de la inseguridad en el transporte de productos y mercancías debido a la proliferación de asaltos y robos a los transportistas, la adopción de políticas destinadas a mejorar el control y a afianzar la seguridad y los servicios policiales podría contribuir a la reducción de los costos incurridos por los exportadores, especialmente en el ámbito de las manufacturas, ya que estas son el blanco más frecuente de este tipo de delincuencia.

Por último, los resultados sobre los efectos de la dotación de factores en las exportaciones de bienes manufacturados revelan los desafíos que deben enfrentar las economías menos desarrolladas a fin de cambiar su patrón de especialización. Para convertirse en un exportador de manufacturas se requiere incrementar la dotación de capital humano. En el caso de muchas naciones en desarrollo, ello implica formular políticas destinadas a mejorar el acceso a todos los niveles de educación y capacitación. En la mayoría de los casos, también supone aumentar el ingreso de los 
quintiles más pobres, así como diseñar e implementar políticas públicas orientadas a crear la infraestructura y los servicios de guarderías y jardines infantiles necesarios como para que las madres pertenecientes a los quintiles de ingreso menos favorecidos puedan incorporarse a la fuerza de trabajo y, por otra parte, a que los infantes tengan acceso desde temprana edad a un ambiente estimulante y determinante de sus habilidades de aprendizaje. Sin embargo, la calidad de la educación parece haberse convertido en la variable más crítica que deberá afrontar la región en el futuro, sobre todo en los países que ya han mostrado avances en esta materia. Ello representa un desafío mayúsculo en términos de políticas públicas, puesto que la tarea de mejorar la educación ha demostrado ser particularmente compleja, incluso en países desarrollados cuya limitación de recursos es muy inferior a la de los países latinoamericanos. En el ámbito de la capacitación, y especialmente de la capacitación en el trabajo, los retos son similares. Al respecto, convendría formular políticas mancomunadas del sector público y del sector privado encaminadas a diseñar e implementar programas que realmente contribuyan al mejoramiento de las ventajas competitivas de los países, las que pueden favorecerse y promoverse mediante el otorgamiento de créditos tributarios a las empresas y sectores productivos que destinen esfuerzos y recursos a la especialización y capacitación de sus trabajadores.

ANEXO

Agregados de Leamer

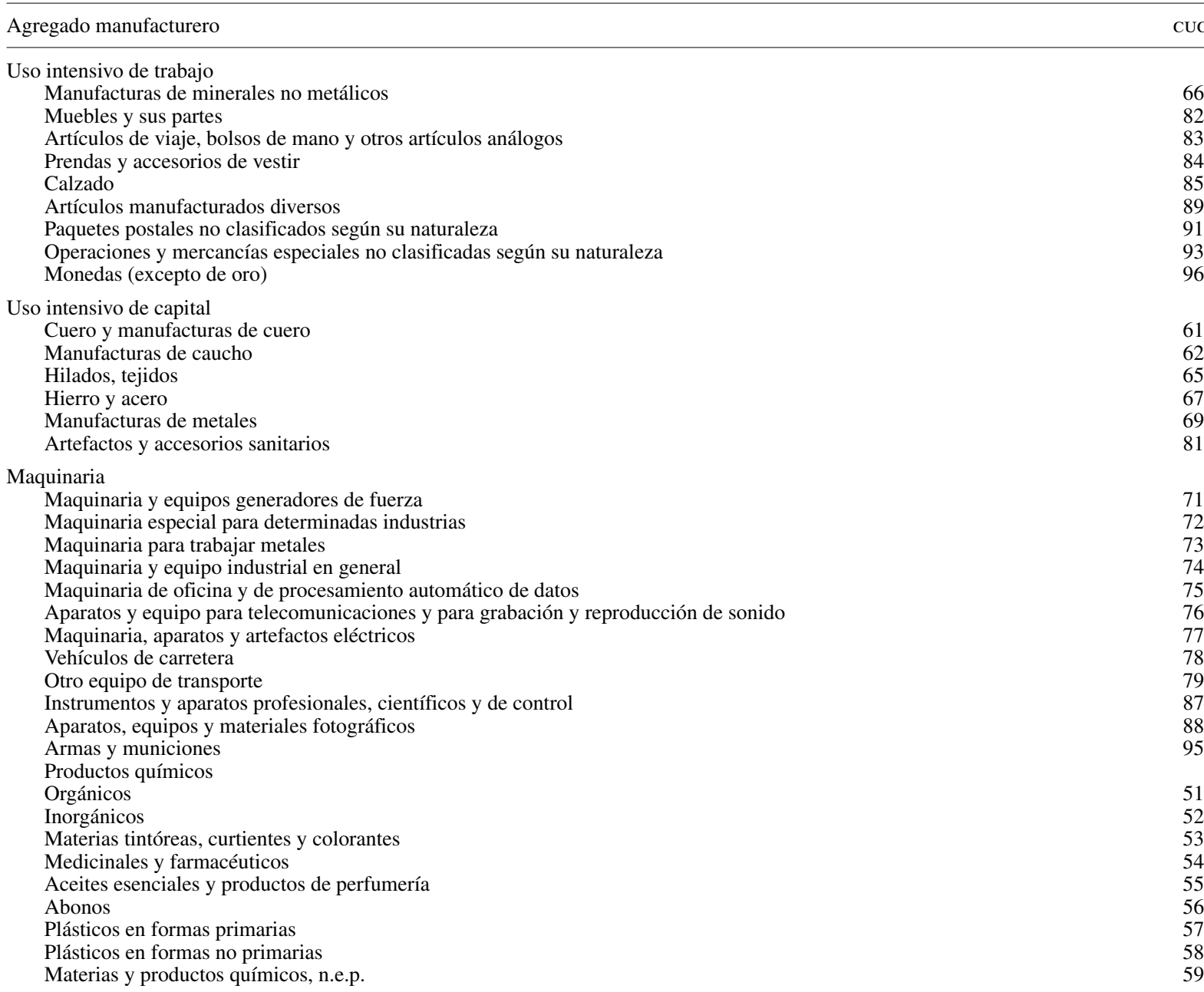

Fuente: E. E. Leamer, "The Heckscher-Ohlin model in theory and practice", Princeton Studies in International Finance, № 77, Princeton, Universidad de Princeton, 1995. 
ANEXO 2

\begin{tabular}{|c|c|c|c|}
\hline Nombre del país & Código del país & Nombre del país & Código del país \\
\hline Argentina & ARG & Kenya & KEN \\
\hline Australia & AUS & República de Corea & KOR \\
\hline Austria & AUT & Sri Lanka & LKA \\
\hline Bélgica & BEL & Marruecos & MAR \\
\hline Bangladesh & BGD & Madagascar & MDG \\
\hline Bolivia (Estado Plurinacional de) & BOL & México & MEX \\
\hline Brasil & BRA & Mali & MLI \\
\hline Canadá & CAN & Mozambique & $\mathrm{MOZ}$ \\
\hline Suiza & $\mathrm{CHE}$ & Mauricio & MUS \\
\hline Chile & CHL & Malawi & MWI \\
\hline Côte d'Ivoire & CIV & Malasia & MYS \\
\hline Camerún & CMR & Nigeria & NGA \\
\hline Colombia & $\mathrm{COL}$ & Nicaragua & NIC \\
\hline Costa Rica & CRI & Países Bajos & NLD \\
\hline Chipre & CYP & Noruega & NOR \\
\hline Dinamarca & DNK & Nueva Zelandia & NZL \\
\hline República Dominicana & DOM & Pakistán & PAK \\
\hline Argelia & $\mathrm{DZA}$ & Panamá & PAN \\
\hline Ecuador & ECU & Perú & PER \\
\hline Egipto & EGY & Filipinas & PHL \\
\hline España & ESP & Portugal & PRT \\
\hline Etiopía & ETH & Paraguay & PRY \\
\hline Finlandia & FIN & Rwanda & RWA \\
\hline Francia & FRA & Senegal & SEN \\
\hline Inglaterra & GBR & Singapur & SGP \\
\hline Alemania & GER & El Salvador & SLV \\
\hline Ghana & GHA & Suecia & SWE \\
\hline Grecia & GRC & Tailandia & THA \\
\hline Guatemala & GTM & Trinidad y Tabago & TTO \\
\hline Guyana & GUY & Túnez & TUN \\
\hline Honduras & HND & Turquía & TUR \\
\hline Indonesia & IDN & Tanzania (Rep. Unida de) & TZA \\
\hline India & IND & Uganda & UGA \\
\hline Irlanda & IRL & Uruguay & URY \\
\hline Irán (Rep. Islámica del) & IRN & Estados Unidos & USA \\
\hline Israel & ISR & Venezuela (Rep. Bolivariana de) & VEN \\
\hline Italia & ITA & Sudáfrica & ZAF \\
\hline Jamaica & JAM & Zambia & $\mathrm{ZMB}$ \\
\hline Jordania & JOR & Zimbabwe & ZWE \\
\hline Japón & JPN & & \\
\hline
\end{tabular}

Fuente: elaboración propia.

ANEXO 3

Principales variables utilizadas

\begin{tabular}{lll}
\hline \multicolumn{1}{c}{ Variables } & \multicolumn{1}{c}{ Descripción } & \multicolumn{1}{c}{ Fuente primaria de datos } \\
\hline X & Exportaciones & COMTRADE \\
DIST & Distancia & CEPII \\
MAR & Salida al mar & CEPII \\
VEC & Contigüidad & CEPII \\
LENGUAJE & Lenguaje & CEPII \\
TIERRA & Tierra arable & Banco Mundial, Indicadores del desarrollo mundial \\
FLABORAL & Fuerza laboral & Banco Mundial, Indicadores del desarrollo mundial \\
R & Tierra arable por trabajador & Banco Mundial, Indicadores del desarrollo mundial \\
ESC & Años de escolaridad & Bosworth y Collins (2003) \\
KL & Capital por trabajador & Bosworth y Collins (2003) \\
APER & Aranceles a las importaciones & Banco Mundial \\
\hline
\end{tabular}

Fuente: Banco Mundial, Centre d'études prospectives et d'informations internationales (CEPII), B. Bosworth y S. M. Collins, "The empirics of growth: an update", Brookings Papers on Economic Activity, vol. 34, N² 2, Washington, D.C., The Brookings Institution, 2003 , y Base de datos estadísticos sobre el comercio de mercaderías (COMTRADE).

Nota: Las variables en las regresiones del modelo se expresan en logaritmos naturales.

a Datos disponibles [en línea] http://go.worldbank.org/LGOXFTV550. 
Álvarez, R. y J.R. Fuentes (2006), "Paths of development, specialization, and natural resources abundance", Documento de trabajo, $\mathrm{N}^{\circ} 383$, Santiago de Chile, Banco Central de Chile, diciembre.

Baier, S.L. y J.H. Bergstrand (2007), "Do free trade agreements actually increase members' international trade?", Journal of International Economics, vol. 71, № 1, Amsterdam, Elsevier.

Blázquez-Lidoy, J., J. Rodríguez y J. Santiso (2006), “¿Ángel o demonio? Los efectos del comercio chino en los países de América Latina", Revista de la CEPAL, Nº 90 (LC/G.2323-P), Santiago de Chile, diciembre.

Blum, B. y A. Goldfarb (2006), "Does the Internet defy the law of gravity?", Journal of International Economics, vol. 70, $\mathrm{N}^{\mathrm{o}} 2$, Amsterdam, Elsevier.

Bosworth, B. y S.M. Collins (2003), "The empirics of growth: an update", Brookings Papers on Economic Activity, vol. 34, № 2, Washington, D.C., The Brookings Institution.

Claro, S. (2006), "Consecuencias para la región de América Latina y el Caribe de la aparición de China en el escenario económico mundial: el caso de Chile", Integración y comercio, vol. 24 Buenos Aires, Instituto para la Integración de América Latina y el Caribe (INTAL)/Banco Interamericano de Desarrollo (BID), enero-junio.

Devlin, R., A. Estevadeordal y A. Rodríguez-Clare (2006), The Emergence of China: Opportunities and Challenges for Latin America and the Caribbean, Washington, D.C., David Rockefeller Center for Latin American Studies/Banco Interamericano de Desarrollo.

Feenstra, R.C., R.E. Lipsey y otros (2004), "World trade flows: 1962 2000”, NBER Working Paper, N N 11040, Cambridge, Massachusetts, National Bureau of Economic Research.

Hanson, G. y C. Xiang (2004), "The home market effect and bilateral trade patterns", American Economic Review, vol. 94, $\mathrm{N}^{\mathbf{0}}$ 4, Nashville, Tennessee, American Economic Association.
Leamer, E.E. (1995), "The Heckscher-Ohlin model in theory and practice", Princeton Studies in International Finance, $\mathrm{N}^{\circ} 77$ Princeton, Universidad de Princeton.

(1987), "Paths of development in the three-good n-factor general equilibrium model", Journal of Political Economy, vol. 95, N ${ }^{\circ} 5$, Chicago, University of Chicago Press.

(1984), Sources of International Comparative Advantage: Theory and Evidence, Cambridge, Massachusetts, The MIT Press.

Lehmann, S., D. Moreno y P. Jaramillo (2007), "China, precio de commodities y desempeño de América Latina: algunos hechos estilizados", Documento de trabajo, № 424, Santiago de Chile, Banco Central de Chile, agosto.

Mesquita Moreira, M. (2007), "Fear of China: is there a future for manufacturing in Latin America?", World Development, vol. 35, $\mathrm{N}^{\mathrm{o}}$ 3, Amsterdam, Elsevier.

Perkins, D.H. y M. Syrquin (1989), "Large countries: the influence of size", Handbook of Development Economics, vol. 2, Amsterdam, Elsevier.

Rosales, O. y K. Kuwayama (2007), "América Latina al encuentro de China e India: perspectivas y desafíos en comercio e inversión", Revista de la CEPAL, No 93 (LC/G.2347-P), Santiago de Chile.

Rose, A. (2004), "Do we really know that the wTo increases trade?", American Economic Review, vol. 94, № 1, Nashville, Tennessee, American Economic Association.

Schott, P.K. (2003), "One size fits all? Heckscher-Ohlin specialization in global production", American Economic Review, vol. 93, №3, Nashville, Tennessee, American Economic Association.

Tinbergen, J. (1962), Shaping the World Economy: Suggestions for an International Economic Policy, Nueva York, The Twentieth Century Fund.

Wacziarg, R. y K. Horn Welch (2003), "Trade liberalization and growth: new evidence", NBER Working Paper, No 10152 , Cambridge, Massachusetts, National Bureau of Economic Research, diciembre. 